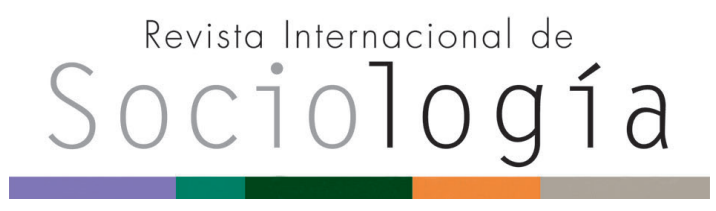

Revista Internacional de Sociología RIS

vol. 75 (1), e055, enero-marzo, 2017, ISSN-L:0034-9712

doi: http://dx.doi.org/10.3989/ris.2017.75.1.15.31

\section{ANTE UNA NUEVA CIVILIDAD URBANA. Capitalismo cognitivo, habitus y gentrificación}

\author{
Jorge Sequera FeRnÁndeZ \\ Universidad Carlos III de Madrid \\ jorge.sequera@uc3m.es \\ ORCID iD: http://orcid.org/0000-0002-8836-425X
}

Cómo citar este artículo / Citation: Sequera Fernández, J. 2017. "Ante una nueva civilidad urbana. Capitalismo cognitivo, habitus y gentrificación". Revista Internacional de Sociología 75 (1): e055. doi: http://dx.doi.org/10.3989/ ris.2017.75.1.15.31

\begin{abstract}
Resumen
La economía del conocimiento se ha convertido en los últimos tiempos en un campo de batalla entre ciudades globales. Actividades relacionadas con la cultura, el conocimiento y los saberes técnicos refuerzan la idea de la necesidad de nodos estratégicos espaciales y la formalización de políticas de atracción a las ciudades a través de la revitalización de los centros urbanos. Para examinar esta puesta en marcha de procesos de gentrificación en el centro de las ciudades contemporáneas, repasaremos la notable importancia que ha adquirido la producción cultural en los últimos tiempos, que ha reportado unas políticas de atracción de trabajadores del conocimiento a los centros metropolitanos fomentando la distinción figurada de una construida "clase creativa". Finalmente, y en contraposición a las tesis vertidas sobre las "clases creativas", estableceremos un diálogo con Bourdieu que nos ayude a discernir si existe una práctica social metropolitana propia de esas nuevas clases medias, o si la yuxtaposición de estas prácticas sociales distintivas son mecanismos que coadyuvan al desplazamiento directo o indirecto de una población no deseada sobre el lugar a revalorizar.
\end{abstract}

\section{Palabras Clave}

Bourdieu; Gentrificación; Producción cultural.

\section{A NEW URBAN CIVILITY Cognitive capitalism, habitus and gentrification}

Copyright: $\odot 2017$ CSIC. Este es un artículo de acceso abierto distribuido bajo los términos de la licencia Creative Commons Attribution (CC BY) España 3.0.

Recibido: 23/02/2015. Aceptado: 18/05/2016 Publicado on line: 07/03/2017

\section{Abstract}

The knowledge economy has recently become a battleground between global cities. Activities related to culture, knowledge and technical knowledge reinforce the idea of the need for strategic spatial hubs and formal policies to attract people to cities by revitalising urban centres. To examine this process of gentrification in the centre of contemporary cities, I will review the remarkable importance attached to cultural production in recent times, which has led to the development of certain policies for attracting knowledge workers to metropolitan centres, thereby promoting the figurative distinction of a constructed "creative class". Finally, I will establish a dialogue with Bourdieu to help us discern whether there is a metropolitan social practice belonging to these new middle classes or whether the juxtaposition of these distinctive social practices is producing mechanisms that are contributing to the direct or indirect displacement of an unwanted population from places of increasing value.

\section{KEYWORDS}

Bourdieu; Cultural production; Gentrification. 


\section{INTRODUCCIÓN}

La economía del conocimiento se ha convertido en las últimas décadas en un campo de disputa entre ciudades contemporáneas. Actividades relacionadas con la cultura, el conocimiento y los saberes técnicos refuerzan la idea de la necesidad de nodos estratégicos espaciales (May 1996) y la formalización de políticas de atracción a través de la revitalización de los centros urbanos. Así, la reconstrucción simbólica de las ciudades está rodeada de conceptos como autenticidad, originalidad o singularidad (Pendlebury et al. 2009), con objeto de poner en venta la cultura y las tradiciones de un determinado lugar (Glaeser et al. 2001). Esta nueva lógica, que combina la importancia de la centralidad espacial y el control de algún recurso específico para extraer rentas de monopolio, atraviesa a la cultura como un tipo especial de mercancía en las ciudades contemporáneas (Lloyd y Clark 2001; Harvey 2007: 418). Se trata del "empresarialismo urbano" (Harvey 1989), en el que los distintos poderes estatales, junto con otras organizaciones y corporaciones privadas, gestionan los cambios de dirección en el desarrollo de la regeneración urbana. Nos referimos a una serie de inversiones públicas y privadas en lugares determinados, que promuevan un espacio urbano para convertirlo en valor de cambio con alto potencial, promocionando un determinado capital simbólico que genere rasgos distintivos y, por tanto, rentas de monopolio (Harvey 2007: 428). Lo que mostraremos a lo largo del texto es que no es solo el suelo o la centralidad en sí misma la que ejerce ese poder especulativo, sino la exclusividad (Fournier 2008; Centner 2008: 208) o cercanía de uso de esa mercancía-cultura.

Tomando como punto de partida las implicaciones del capitalismo posfordista, esto es, un modo de regulación flexible que articula elementos geográficos, sociales y tecnológicos distintos al fordismo (Boyer 1986), podemos observar las implicaciones socioespaciales de este modelo de acumulación dentro de las ciudades en sus efectos sobre procesos como la gentrificación (Carman 2006; Zukin 1995; Butler 1997, 2002; Sequera y Janoschka 2015), mediante la articulación de los nuevos nichos de mercado (cultural, del conocimiento), las nuevas formas de empleabilidad, su repercusión en los consumos y los estilos de vida en los centros de las ciudades globales. Si en la etapa anterior el consumo dependía de la empresa pública, de la producción en masa y de una fuerte ciudadanía social y laboral (Alonso 2004), a partir de los ochenta del siglo XX aparecen con fuerza los flujos informacionales, los derechos intelectuales, las tecnologías, la cultura como recurso y una fuerte actividad financiera. Este paradigma viene aparejado a dinámicas de fragmentación de la ciudadanía, de individualización y de globalización y, por supuesto, a nuevos estilos de vida y consumo distintivos que se reflejan en la manera de entender la ciudad, que también se ve fragmentada y segregada socialmente por las distintas identidades emergentes. Para examinar esta puesta en marcha de un modelo económico y urbano, que además promociona procesos de gentrificación en el centro de las ciudades contemporáneas (Deutsche y Ryan 1984), repasaremos en primer lugar la notable importancia que ha adquirido la producción cultural en los últimos tiempos, que ha reportado unas políticas de atracción de trabajadores del conocimiento a los centros metropolitanos fomentando la distinción figurada de una construida "clase creativa".

Los gestores de las ciudades de todo el mundo parecen haberse enamorado de la idea de la "ciudad creativa" (Florida 2005) intentando, como veremos, atraer a la llamada "clase creativa" (artistas, intelectuales, gente del espectáculo, diseñadores, etc.) a vecindarios en proceso de regeneración, bajo la siguiente premisa: las ciudades contemporáneas más prósperas serán aquellas que sepan combinar acertadamente unos estilos de vida alternativos basados en la cultura y el ocio como elementos primordiales en su propia configuración. En tanto que estrategia urbana generalizada, plantearemos en segundo lugar que las políticas urbanas que siguen estas tesis (Florida 2003) están tratando de promover procesos de gentrificación en torno a la noción de clase creativa (Scott 2004; 2006; Markusen 2006; Peck 2005; Krätke 2010; Boschma y Fritsch 2009; Lang y Danielsen 2005) y son el correlato de un sistema de prácticas que condicionan el espacio social y sus usos. Finalmente, y en contraposición a las tesis vertidas sobre las "clases creativas", estableceremos un diálogo con Bourdieu que nos ayude a discernir si existe una práctica social metropolitana propia de esas nuevas clases medias o si la yuxtaposición de estas prácticas sociales distintivas son mecanismos que coadyuvan al desplazamiento directo o indirecto (Marcuse 1985; Slater 2009) de una población no deseada sobre el lugar a revalorizar.

\section{LA PRODUCCIÓN EN (DE) LA CIUDAD CREATIVA}

Una de las estrategias urbanas generalizadas en la era del neoliberalismo es la construcción simbólica de la "ciudad creativa" (Florida 2010). Es común oír que gestores públicos retraten la clave del éxito como una combinación entre un clima empresarial sólido y un clima personal afectivo apropiado, con el que poder retener a esta "clase creativa". Una vez desmantelada la fábrica como paradigma del centro de trabajo y con la oficina en fase de profundos cambios relacionados con la flexibilidad social y laboral (Bauman 2007), emergen las propias personas como recurso natural, como principal fuente de creatividad. La lógica, por tanto, siguiendo con el argumento de concentración de las ciudades globales (Sassen 2001; Castells 2001), sería la de atraer este 
tipo de potencias creativas al centro de las ciudades. Pero este tipo de políticas públicas tiene como consecuencia procesos de gentrificación, cuya norma parece ser el asalto de las clases medias y mediasaltas a este tipo de escenarios.

En este contexto, la economía cultural necesita que los centros urbanos tengan entornos proactivos, enriquecedores, para el desarrollo de las nuevas clases medias y la ciudad creativa. El rol que ha adquirido la producción cultural va unido a su espectacularización y el aprovechamiento del fuerte tirón de la cultura para "redecorar" ciertas partes degradadas del centro de la ciudad (García y Sequera 2013). Por tanto, esta economía urbana cultural trata claramente de establecer una serie de tramas entre los sujetos del territorio, la ciudad, la cultura y la política. Así, las nuevas formas de gobernanza urbana que los poderes públicos utilizan para capturar con más eficacia formas de creatividad social, contienen nuevas formas relacionales, tanto en el ámbito laboral como en el económico o social. Teniendo en consideración que la llamada economía creativa (Méndez et al. 2012), se refiere tanto a la producción de bienes simbólicos (con un marcado carácter industrial y de consumo de masas) como a las actividades culturales más tradicionales, las industrias creativas son variaciones instrumentales que tienden a convertir a la industria cultural (Garnham 2005) en un sector económico capaz de soportar su propio desarrollo socioeconómico, y a su vez como una estrategia de desarrollo local (Navarro y Guerrero 2010). Es lo que se ha denominado como "fábricas de la cultura" (Carrillo 2007), que mediante sus infraestructuras y grandes contenedores de arte contemporáneo se perfilan como la nueva industria empresarial de la ciudad.

En el nuevo campo de la creatividad y la innovación, estos mismos conceptos se convierten en los nuevos engranajes de la "ciudad fábrica". La ciudad como un gran museo ${ }^{1}$ parece la pretensión de los nuevos desarrollos "culturalistas" de los poderes públicos, donde los habitantes se conviertan en espectadores, en turistas universales. Es decir, un dispositivo del espectáculo como herramienta de la nueva civilidad del ciudadano urbano. En este contexto, lo que nos interesa por tanto es comprender la instrumentalización del concepto de cultura en la modificación del lugar y sus sentidos (Carman 2006; Bianchini y Parkinson 1993; Mommaas 2004). Entendiendo entonces que se necesita innovación para confeccionar un ciclo económico que no solo siga las pautas necesidad-demanda sino que las coproduzca, qué mejor lugar que las ciudades contemporáneas, donde emerge lo que es considerado transgresor, el sobreestímulo, lo alternativo (Shaw 2005), para que funcione de brainstorming colectivo y así poder cosificarlo. No parece existir otro sitio mejor que la metrópolis para que se desarrollen los procesos cul- turales innovadores y se conviertan con el tiempo en artes mercantilizados y elementos de distinción. De este modo, asistimos a una explotación constante de los conocimientos para desarrollar más productos y servicios.

Al respecto, Yúdice (2002) afirma que en el capitalismo contemporáneo la cultura es un recurso, y entre sus múltiples usos está el de convertirse en proveedora de turismo (Fainstein y Gladstone 1999; Gotham 2005), de desarrollo urbano y de crecimiento económico, siguiendo la lógica mimética de las ciudades globales mediante su financiación y fomento (Yproductions 2009: 114). De esta forma, la innovación es el exponente fundamental de esta maquinaria urbana, economizando la creatividad social (Jacobs 1969; Hall 1998). La instrumentalización que hace la Administración Pública del concepto de innovación es la resultante de una gestión urbana que busca en esta industria cultural el crecimiento económico de una ciudad: desarrollo local, regeneración urbana, turismo cultural, etcétera. La creatividad parece haberse convertido por tanto, en la etiqueta clave que impulsa el interés en este tipo de mercado. Esto nos lleva a la cultura de la innovación (Yproductions op. cit.: 136), en la que una serie de dispositivos (con distintas formas, discursos, instituciones, espacios, lugares, gentes) pretenden generar ese clima de innovación.

Parece por tanto evidente que el proceso de llenado cultural, artístico, científico u ocioso que se desarrolla en las ciudades, convierte los centros antiguos en parques temáticos de la cultura y en escenario de nuevas civilidades. Así pues, en los procesos de gentrificación, las clases medias y altas son dirigidas al interior de los barrios, caracterizándolos y dotando al lugar de "unas dosis controladas y controlables de culturalismo, en definitiva, un nuevo sabor local que atraiga a potenciales clientes" (Delgado 2007). Las industrias culturales, generadoras de productos para el consumo masivo y homogeinizadoras del gusto, son las encargadas de este modelo; y su contenido cultural, como particular tipo de mercancía, consiste en una serie de actividades que "tienden a definir el contenido cultural, artístico, de moda, gustos y consumo estándar" (Domínguez 2008: 9).

\section{LA TRAMA DE LA CLASE CREATIVA}

"La creative class evidencia la esquizofrenia del trabajador posfordista, esto es, el hecho de ser al mismo tiempo trabajo y capital". De Nicola et al. 2008: 51.

Como estamos viendo, la construcción de una narrativa de la creatividad (Méndez et al. 2012: 28) ha recibido un impulso por parte de las instituciones políticas locales, nacionales e internacionales en un intento por dar coherencia a todo un discurso acerca del potencial del capitalismo cultural-cognitivo (Scott 2007; 2008). Si definimos a la economía creativa 
como aquella que basa su producción en la creación de bienes simbólicos, que dependen de derechos de propiedad y que se dirigen a un mercado muy amplio (Unctad 2008), observamos que el trabajo cultural, cognitivo, emerge como uno de los más representativos en las nuevas formas de producción. Y el trabajador del conocimiento (De Nicola et al. 2008) es el sujeto clave en esta profesionalización e intelectualización de la fuerza de trabajo. Según afirma De Giorgi (2006: 128), el conocimiento se ha transformado en fuerza productiva inmediata: donde antes las puertas de la fábrica determinaban el espacio disciplinario de control de las fuerzas de trabajo, ahora se transita hacia un espacio posfordista, que allana la rocosidad de esos espacios de vida delimitados, haciendo controlables los flujos de productividad social. En la actualidad, por tanto, el plusvalor puede ser obtenido prácticamente de todo lo que ocurre en la vida. Este potencial que desarrollan los knowledge workers -o el cognitariado, o las creative class- es puesto al servicio de la acumulación y reproducción del sistema, definiendo así el paradigma del capitalismo cognitivo como la producción de conocimiento y su gestión empresarial, en búsqueda constante de innovaciones que puedan convertirse en diferenciación para su comercialización.

Conscientes de la fuerza de este tipo de trabajadores, el discurso neoliberal ha apostado por la creación de una nomenclatura elitista que articule distinción, legitimación y exclusividad espacial de estas "clases". De esta forma aparecen con fuerza conceptos como el de creative class ${ }^{2}$ de Florida (2010), que los define como el grupo de personas con intereses, formas de pensar y comportamientos comunes, determinados fundamentalmente por la función económica, no solo por su condición económica. Florida insiste en la descripción de esta particular construcción de la clase creativa, a partir de adjetivaciones como la individualidad o la meritocracia ${ }^{3}$, al mismo tiempo que compensa estas razones eminentemente liberales con las de "diversidad y apertura". Sin embargo, tras su trabajo encontramos pistas para poder observar un barrio gentrificado, mediante esos estilos de vida que al propiciarse en un entorno concreto pueden hacer cambiar un barrio (Blockland y Van Eijk 2010). Según advierte críticamente McGuigan (2009: 298), Florida no investiga acerca de la política cultural, sino que busca la articulación entre la economía neoliberal y la cultura cool.

En este pretendido salto hacia una sociedad postindustrial, los principales enclaves mundiales de los países desarrollados han pasado de producir bienes a producir servicios. En este escenario, la clase trabajadora -afirmará Florida (2010: 49) - ha disminuido y la clase de servicios sigue siendo mayor, mientras que la clase creativa es dominante en términos de riqueza y de ingresos. De esta manera, introduce una de las trampas, siendo realmente uno de los grupos sociolaborales que sufre mayor flexibilidad y precariedad laboral: al depender de proyectos, vivir "al día”, estar atravesado por vínculos débiles debido a su constante movilidad, y representar la empresarialidad de sí mismo, entre otros factores. Así, observamos "la preeminencia del trabajador del conocimiento" como sujeto activo de estas políticas neoliberales del trabajo. Como plantea Lorey (2008: 73) al respecto de este tipo de subjetividades, las condiciones de trabajo y de vida de estos trabajadores, en muchos casos alternativa, favorece las formas flexibles y precarias que el mercado neoliberal está suscitando. Estas formas de vida van aparejadas al hecho del agenciamiento como herramienta del individuo, que sobrepasa la estructura, pero que es subsumido otra vez bajo nuevas formas de gubernamentalidad neoliberal (Sequera 2014). La apariencia de la capacidad de las personas a actuar de forma independiente y tomar sus decisiones libremente dentro de un determinado campo que estará condicionado por la estructura y a la vez determinando la estructura, enmarcadas siempre en la productividad de la vida misma, genera como resultado un habitante de las ciudades reorganizado bajo la lógica de la precariedad vital (Gill y Pratt 2008; Menger 1999; Storper y Scott 2009): trabajo precario en sí, bajo proyectos, temporalidad, constante movilidad, indiferenciación del tiempo de trabajo y de ocio, etcétera.

En definitiva, es la flexibilidad de las relaciones sociales que quizá se liberaron desde aquel Mayo del 68 la que, reorganizada desde el capital, se convierte en flexibilidad en todos los aspectos de la vida, subsumiendo la vida al trabajo. Esto ha generado necesariamente nuevas formas de trabajo flexibles del capitalismo cognitivo, fomentadas sobre todo por la economía urbana en las ciudades globales. Esta flexibilidad incluye la precarización, y la pérdida de los límites entre el tiempo de trabajo y el libre. Pero esa libertad y autonomía a la que se rendía culto como el adalid de la liberación es convertida en recurso del propio devenir del capitalismo cognitivo. Estas nuevas formas de vida urbanas, que constituyen nuevas subjetividades, están a disposición del desarrollo de la economía capitalista y sus procesos de acumulación. Lo cierto es que una serie de nuevas profesiones, en muchos casos independientes o autónomas, se han introducido al mercado, externalizando ciertos servicios y formalizando al sujeto como empresa. De hecho, "las ideas de autonomía y libertad están constitutivamente conectadas con los modos hegemónicos de subjetivación en las sociedades capitalistas occidentales" (Lorey 2008: 58). En última instancia, el éxodo de la fábrica y la búsqueda de autonomía han sido reconvertidos en la consentida precariedad sociolaboral de estos sujetos.

Entonces, ¿qué es realmente la creative class? Para que su tesis adquiera legitimidad, Florida (2010: 47) lanza un último alegato, refiriéndose a la clase 
como el grupo de personas definido por la función económica, es decir, por su trabajo, en lugar de articular sus estilos de vida o consumo con su nivel de ingresos. Así, nos encontramos ante una compleja y controvertida descripción del trabajador cognitivo (Hall 2007: 208), tanto por su ubicación en el clásico sistema de clases vertical como por su dimensión temporal, precaria y de constante innovación y formación. Esta tesitura resulta delicada debido a la dirección que toman este tipo de trabajos, respecto de sus más cercanas figuras (el asalariado, la clase obrera), de sus particulares estilos de vida (clase media) y de su no reconocimiento claro como "trabajador". Dentro de este nuevo perfil que se ha consolidado en la sociedad informacional, el reto consiste en observar cómo se posiciona, si como un sector de élite, separado de obreros y precarios, o como sujetos del cambio en busca de reconocimiento en la sociedad (De Nicola et al. 2008). En este proceso urbano de la centralización de la alta profesionalización, puede emerger -como ya hemos expuestouna fuerte polarización social debido a lo restrictivo del estilo de vida de estos trabajadores. Al fin y al cabo, parece ser un tipo de (nueva) clase media (urbana), innovadora y progresista. Esto nos reporta al eterno dilema "estructura frente a agencia": estas nuevas clases medias son las que hacen del centro de las ciudades un nuevo lugar (Ley 1996; 2003), mediante sus nuevos patrones de consumo, pero al mismo tiempo, es la agenda neoliberal la que los reclama con medidas políticas concretas (Smith 2008; 1979; 1982), como "ejemplificadores" sociales para la propagación de la gentrificación.

Porque detrás de estas estrategias de "hipsterización" (Sequera 2013) hallamos políticas de atracción. Ciudades luchando por acaparar ese talento, que mejore no solo la economía urbana, sino el entorno urbano a partir de mecanismos de exclusión; como critica Peck (2005: 740) en Struggling with the creative class. Toda una generación de planeadores urbanos progresistas confían en este dogma como una solución integral para las ciudades, configurando una agenda política urbana determinada por estrategias que privilegian a ciertos actores sociales frente a otros. Estos pretenden mezclar el elitismo cosmopolita con la universalización del pop; el hedonismo con la responsabilidad; y la cultura alternativa con la economía urbana tradicional (Ibíd.: 741). En un intento por revitalizar los barrios, pretenden crear entornos trendys aprovechando ese tipo de lugares cargados de estímulos. La razón parece ser esa búsqueda del fortalecimiento de la economía del conocimiento, que entiende como necesario un clima urbano propicio para este tipo de trabajadores creativos. En realidad, tal como propone Harvey (1989), la idea es volver a poner a la ciudad en el centro del consumo, y para ello tiene que aparecer como innovadora, estimulante, creativa y segura para vivir o visitar. En otras palabras, estamos ante un dispositivo neoliberal que utiliza los mecanismos culturales urbanos con objetivos competitivos y economicistas (Buck et al. 2005; Musterd y Murie 2010) para generar lo que Peck (Ibíd.: 764) ha llamado "gentrification-friendly", es decir, políticas que tratan de dar una cara amable a estos procesos de desplazamiento. Donde otros críticos observan las consecuencias negativas de la gentrificación (Janoschka y Sequera 2016), como la subida del precio de la vivienda o las distintas formas de desplazamiento, Florida (2003) encuentra un indicador de lo atractivo que es un lugar, la demanda real de un espacio urbano concreto y, por supuesto, la simple sustitución de la clase trabajadora por un nuevo perfil.

Finalmente, proponemos que los estilos de vida, como componente definitorio de la clase social que incluye una actualización del término marxista derivado de la posición económica-, permiten que entren en juego debates sobre las "culturas del gusto", el "consumo conspicuo" o la "estética". Se trata entonces no solo de una clase social en cuanto al factor transversal derivado de la posición en la cadena de producción, sino en cuanto a la construcción de una identidad basada en el consumo como una forma de inversión, como símbolo y medio de expresión; es decir, la compra de una identidad concreta. Así, las clásicas categorías sociológicas que venían determinadas por la posición del sujeto respecto de los medios de producción deben ser necesariamente complementadas por otras categorías tales como el estatus social o los distintos capitales (simbólico, relacional, social, etc.) a los que nos tiene acostumbrados el análisis bourdieano, tal como veremos en el siguiente apartado.

\section{ESTILOS DE VIDA Y CONSUMO DISTINTIVO EN LOS PROCESOS DE GENTRIFICACIÓN. UN DIÁLOGO CON BOURDIEU}

Butler (2002) y Butler y Robson (2001) compararon seis barrios de Londres para buscar los distintos significados que los residentes potenciales hacían del barrio y los motivos por los que se asentaron allí, es decir, cómo los gentrificadores se comportan para asegurar su hegemonía en los sitios donde se han afincado. Así, demostraron las dinámicas de extracción de capital cultural, económico y social de un área determinada, con su consecuente gentrificación, y observaron lo que denominaron como "habitus metropolitano" (metropolitan habitus). Buscaban en ello no solo la explicación del proceso, sino saber cómo se producen espacialmente las distintas inclinaciones de los grupos entrevistados, para lo que sugirieron que el habitus metropolitano debía ser un recurso conceptual mejor explicado, que podría revelar diferencias subyacentes entre periferia y centro, 0 entre centro y centro, dependiendo del uso del mismo. El estudio que presentó Butler aportó elementos clave para entender la atracción de este habitus me- 
tropolitano fuertemente asociado con la ciudad global y sus emergentes connotaciones culturales, que vinculaba al nacimiento de sentimientos de localismo y autosegregación dentro de las clases medias. Su tesis fundamental se basó en la articulación del consumo cultural en el centro (museos, galería, teatros, restaurantes, etc.) y las zonas residenciales gentrificadas en los alrededores de este centro. Lo define, aplicado a cada caso, como la creación de un marketing espacial que mercantiliza una zona rehabilitada.

Así, a estas nuevas formas de las clases medias las divide en corporate undistinctives, liberal ascetics y postmoderns (Butler 2002: 7), una composición realizada originariamente por Savage et al. (1992). Los primeros serían burócratas y administradores, con patrones de consumo exclusivo. Los liberal ascetics, profesionales de la educación, el bienestar o la salud, con un consumo cultural alto. Los posmodernos serían profesionales del sector privado (servicios financieros, publicidad, etc.), marcadamente hedonistas. Por su parte, Featherstone (1991: 34) también se refiere a estos últimos como intelectuales y especialistas en producción simbólica; mientras que Bourdieu (1988) los llamaba los "nuevos intermediarios culturales", aquellos que producen servicios y bienes culturales, constituidos a partes iguales por recursos económicos y culturales.

La emergencia de estas variantes de las clases medias definidas espacialmente ha tenido su énfasis en la búsqueda del sentido de identidad, basado en la estética, los estilos de vida, el consumo y el gusto, todos estos asociados a los centros metropolitanos y la distinción que propician a sus residentes. Este enfoque resulta del todo prometedor para los estudios sobre gentrificación contemporáneos. Sin embargo, Bridge (1995) nos advierte acertadamente de que la mayoría de los efectos de la constitución de clase ocurren fuera de los barrios gentrificados (en la división del trabajo y en las relaciones laborales, en el centro de trabajo, en la posición de clase heredada) o antes de que el proceso haya tenido lugar (socialización a través de un determinado estilo de vida o de gusto concreto). Por lo tanto, considera que la adscripción a unas prácticas de clase es previa y no una mera reacción contra un ambiente de clase obrera o un afán por distinguirse. De este modo, consideramos que un diálogo con Bourdieu y sus complejas construcciones del habitus nos ayudarán a arrojar luz, por un lado, sobre la relación entre prácticas distintivas, gentrificación y los sistemas de disposiciones que constituirían un posible habitus metropolitano; y por otro, sobre la disputa por la hegemonización del lugar por las nuevas clases medias, que desplazan a otras posibles.

Cuando hablamos de consumo y estilos de vida ingresamos en el complejo terreno del gusto. Definiremos el gusto como "la propensión y actitud para la apropiación (material y simbólica) de una clase determinada de objetos o prácticas enclasadas y enclasantes, es la forma generalizada que se encuentra en la base del estilo de vida, conjunto unitario de preferencias distintivas, que expresan, en la lógica específica de cada uno de los subespacios simbólicos -mobiliario, vestido, lenguaje o hexis corporalla misma intención expresiva" (Bourdieu 1988, citado en Alonso 2005: 8). Según Bourdieu (2010: 231), "los gustos en la cultura no son naturales, sino que son productos de la educación. Todas las prácticas culturales y las preferencias están ligadas al nivel de instrucción y con el origen social". Así relaciona los gustos con las disposiciones privilegiadas de clase, haciendo que la cultura funcione como un tipo de capital (el cultural), que está desigualmente distribuido al otorgar beneficios de distinción. $Y$ añade: "Es lo que hace que el arte y el consumo artístico estén llamados a cumplir, se quiera o no, se sepa o no, una función social de legitimación de las diferencias sociales" (Ibíd.: 239).

Siguiendo con las interpretaciones de Bourdieu sobre las prácticas de los actores sociales con relación al arte y la cultura en un campus concreto, entiende que estos, movidos por intereses (no necesariamente en un sentido negativo ni economicista) hacen emerger la búsqueda de la dominación; una lucha en la que cada grupo social intenta dominar el campo, mediante el uso de los distintos capitales que configuran el habitus, es decir, mediante la materialización de la posición social y su puesta en práctica bajo esquemas de percepción-acción propios del grupo. Por eso, precisamente, los procesos de ruptura cultural pueden suponer un cambio de modelo de ese campo. De este modo, y como advertíamos, nuevas disposiciones se instauran lentamente mediante la "práctica hecha posición social", paso siguiente que nos descubre Bourdieu (2000a) a la hora de explicar concisamente el concepto de "habitus":

\footnotetext{
"Sistemas de disposiciones duraderas, estructuras estructuradas predispuestas a funcionar como estructuras estructurantes, es decir, en tanto que principios de generación y de estructuración de prácticas y representaciones" (P. 256).
}

En consecuencia, para comprender los procesos de gentrificación simbólica (Janoschka, Sequera y Salinas: 2014: 1249) debemos resaltar la importancia de los distintos capitales en juego, con especial atención al capital cultural y al rol de la cultura en la reproducción de la desigualdad. La razón para esto es la lógica articulación de dicho capital cultural con su propio campo cultural y la formación y consolidación espacial de grupos sociales. No debemos olvidar, como nos presenta Bourdieu (1985: 206), que el capital cultural también sufre procesos de circulación y acumulación, por lo que puede ser uno de los dispositivos gentrificadores más eficientes, como elemento sistemático de desigualdad y segregación ${ }^{4}$. Siguiendo esta premisa, estos sistemas de disposi- 
ciones que componen el habitus se presentarán en los procesos de subjetivación que se materializan en el sentido del lugar, es decir, a través del campus (como las instituciones culturales, el consumo, etc.), correspondiéndose con la lógica bourdieana de interiorización de la exterioridad. Todas estas categorías forman parte de unas condiciones de existencia propias de un barrio concreto, que también sufre las externalidades de la estructura, es decir, de las políticas urbanas que se aplican al mismo. De este modo, las prácticas de consumo y el tipo de relaciones sociales que se generan en su campo son atravesados por una reorganización simbólica y física tras un proceso de transformación urbana. Entonces, si el lugar es modificado, probablemente las prácticas sociales que se desarrollen en su interior sean reconfiguradas también, sufriendo muchos de estos desajustes entre su habitus pasado y el campus actual, esto es, la histéresis del habitus (1993).

En este sentido, la identidad de un grupo es relacionada por Augé (1992: 51) con la identidad del lugar (lo que lo funda, lo une), y el grupo (reconfigurado o no) defenderá la identidad que se conforme para que conserve su sentido. Se trata por tanto de la constitución de lugares mediante la organización del espacio, una práctica social habitual en un territorio. Según este autor (ibíd.: 58), "todos son lugares cuyo análisis tiene sentido porque fueron cargados de sentido, y cada nuevo recorrido, cada reiteración ritual refuerza y confirma su necesidad". Se produce lugar e identidad cultural en la interacción, a través de procesos de hibridación de distintas escalas (Soja 2008: 304), de la más global a la más local, y viceversa. De esta manera se ponen en juego las distintas posiciones, articulando nuevas relaciones de autoridad y de prácticas políticas, en las que se renegocian los sentidos del campo entre lo que se considera hegemónico (centro) y lo que no (margen).

En suma, todas estas consideraciones teóricas previas se pueden aplicar acertadamente a la interpretación de un texto que Bourdieu (1999) Ilamó "efectos de lugar", en el que relaciona de forma precisa el espacio físico con el espacio social. El espacio social -nos dirá- se caracteriza por su posición relativa frente a otros lugares y por la distancia con ellos, es decir, como "estructura de yuxtaposición de posiciones sociales" (P. 120) que genera exclusión mutua o distinción. El espacio habitado simboliza el espacio social, que se traduce en espacio físico, lugar donde se expresa la posición del sujeto a partir de la distancia con otros y la proximidad que adquiere a través del pago o del usufructo de determinadas propiedades o a través del consumo ostentoso del espacio (ibíd.). De este modo, el espacio, los lugares y los sitios son el objeto del conflicto, por la obtención de beneficios tales como las "ganancias de localización", que no son otra cosa que lo que Harvey ha definido tantas veces como "renta mo- nopolista". Se trata de estos espacios construidos, físicos, arquitectónicos que tienen la capacidad de producir lugares bajo la violencia simbólica de la segregación residencial o de uso, o el desplazamiento directo o indirecto (Janoschka y Sequera 2016) de distintos habitus. Estas ganancias de localización pueden definirse desde dos perspectivas: las rentas de situación, es decir, la situación privilegiada junto a agentes o bienes escasos o deseables; o la posición o rango, esto es, las ganancias simbólicas, que generan distinción porque se vinculan al monopolio de una propiedad distintiva. He aquí la aportación definitiva que este texto de Bourdieu realiza al estudio de la gentrificación, es decir, tener la capacidad de dominio del espacio por parte de ciertos sujetos privilegiados, poniendo distancia (física o simbólica) a lo indeseable y apropiándose de aquello que facilita la acumulación de capital social sobre el propio lugar (Bourdieu op. cit.: 122).

Tanto el habitar contribuye a formar el habitus, como el habitus construye el hábitat a través de las inclinaciones en los usos sociales que se hacen de él (Bourdieu 1999). Es aquí donde su definición del habitus de clase adquiere todo su poder, recogiendo la tesis de Elias (2001), por la que los gustos estéticos de las clases dominantes se han tratado de reproducir históricamente en los demás grupos sociales, sin olvidar que las clases populares también construyen una estética del gusto propia y que está siendo reapropiada por ciertas clases medias urbanas. Esto es lo que Alonso (2005) llama "la materialización de la clase". Así, se genera un tipo de consumo distinguido, que utiliza obras, objetos, tipos de vivienda o barrios, que se revalorizan bajo un tipo muy particular de "gusto", seña distintiva de estas clases profesionales urbanas, como consumidores de "signos" y "formas" (Lash y Urry 1994). Como ejemplo, Podmore (1998: 289), siguiendo el estudio de Zukin (1989) sobre el Soho neoyorquino, analizó lo que llamaba el "Síndrome Soho", por el que los lofts (edificios fabriles reconvertidos en estudios y viviendas diáfanas) se convirtieron en un mercado de la vivienda con enormes beneficios para promotores, a partir de su mercantilización como modo de vida identitario. Esta autora describió con el nombre de "habitus loft" a las disposiciones de sus compradores y residentes marcadas por patrones del gusto, prácticas sociales, estilos de vida, de vivienda y uso del espacio.

Por tanto, en nuestra explicación sobre los dispositivos de gentrificación (Sequera y Janoschka 2015) también debemos tener en cuenta los estilos de vida (Bourdieu 2000b), que se deben observar a través de la capacidad para producir unas prácticas y la capacidad de apreciarlas y, por tanto, de diferenciarlas. Estos estilos de vida son producidos y reproducidos por el habitus, entendido como identidad social que se define sobre la diferencia. De esta manera, podemos llegar a distinguir entre consumos distinguidos 
y vulgares. Así "una clase se define por su ser percibido tanto como por su ser; por su consumo -que no tiene necesidad de ser ostentoso para ser simbólico- tanto como por su posición en las relaciones de producción" (Bourdieu 1988: 494). Los estilos de vida son la materialización de las prácticas sociales de clase, que se concretan -como ya señalamos-en capital cultural (titulaciones y credenciales), capital social (conjunto de relaciones socialmente útiles) y capital simbólico (signos, rituales y prácticas de respetabilidad social) (Alonso 2005). De esta forma se consigue complejizar el análisis, articulando cultura y economía bajo el concepto mismo de consumo. Un consumo que se define por los campos producidos y reproducidos culturalmente, desde el gusto hecho necesidad de estas nuevas clases medias hasta la necesidad hecha gusto de las populares.

Resulta importante aclarar que nos referimos a una necesidad ya no entendida solo como carencia (o instrumentalidad), sino como deseo. Necesidad como un tipo de relación social (Alonso 1986) que tiene un carácter histórico basado en el modo de producción de cada sociedad concreta y dentro de esta en su propia división del trabajo. Es por esto que en el capitalismo contemporáneo queda definida la necesidad dentro de la dimensión del consumo - desigual- que "reproduce en el orden de la distribución el orden de la diferencia" (Alonso 1986). Este acceso al consumo deriva directamente de la desigualdad del poder adquisitivo y para ello se crean, más allá de los objetos relacionados con resolver la necesidad como carencia, productos "superfluos" que generen estatus en su poseedor. Por tanto, según la construcción socioespacial que se haga de las necesidades en el consumo, obtendremos el lugar que las necesidades-deseos ocupan en el hecho social.

Por otro lado, como nos plantean Lash y Urry (1994), la jerarquización de los estilos de vida y modos de consumo se yuxtaponen globalmente y las nuevas clases medias urbanas (con un fuerte capital social y simbólico) adquieren un mismo sentido del gusto de una ciudad global a otra y de esta a una ciudad que pretende serlo. Además, el espacio social es pluridimensional, coproducido por campos autónomos, aunque subordinados (Bourdieu 1984/1985) al campo de la producción económica, donde cada subespacio estará a su vez ocupado por posiciones dominadas y dominantes en constante lucha, sin conformarse necesariamente como antagónicos (íbid.: 221). Siguiendo a Featherstone (1995: 110), "las ciudades del mundo son los sitios en los que se advierte la yuxtaposición del rico y el pobre, los profesionales de la nueva clase media y los homeless, junto con una variedad de otras identificaciones tradicionales, étnicas y de clase". De hecho, según las interpretaciones que este autor realiza de Elias (1990 [1987]), la situación jerárquica de ciertos grupos dominantes puede ser capaz de colonizar a los demás grupos sociales e incorporarlos a un patrón de conducta único. Sin embargo, no debemos caer en que estas condiciones objetivas desencadenen directamente una nueva serie de prácticas (Alonso 2005), sino que habrá una disputa por el nuevo sentido del campo. Si esto lo materializamos nuevamente en el lugar, en el espacio físico habitado, podremos observar distintas clases urbanas conviviendo en un mismo espacio, como parte de dicho dispositivo gentrificador, es decir, donde no necesariamente unas deben imponerse a las otras, sino realizar distintos usos y servir de distintos modos al campo económico, esto es, a este capitalismo multiescalar.

Como ya señalamos, frente al modelo de consumo del primer fordismo se construye un consumo distinguido, que utiliza la cultura, el arte y la creatividad para generar el estilo de una clase profesional urbana emergente, que transforma en signos de distinción un gusto social distinto a la serialización del proceso anterior. En la situación actual, las dinámicas de fragmentación y segmentación del consumo posfordista erigen en la metrópolis una figura esencial: las clases medias y medias-altas profesionalizadas, que rompen con el consumo de masas en busca de un nuevo consumo ostentoso y diferenciado de la burguesía tradicional. Estas formas de consumo privativas, cuyo carácter excluyente es precisamente el que convierte al producto en comercializable, representa la desigualdad en la vida cotidiana de las urbes. Sobre esta base -entendemos- se genera reconocimiento social y estatus y, por ende, desigualdad y segregación urbana. Esta lógica no es otra que la perseguida por el neoliberalismo urbano y, en nuestro caso, esto es, el uso de la mercancía como eje reproductor en todas las parcelas de lo social.

\section{A MODO DE CONCLUSIÓN}

Los impactos de la globalización en el espacio urbano y en la vida cotidiana de sus habitantes vienen acompañados de un aumento de la polarización en determinadas áreas urbanas. Estamos, por tanto, ante una nueva organización espacial en (de) las ciudades que depende de un capitalismo cognitivo, demandante de innovación constante (creación de necesidades y demandas), que parece haber encontrado su lugar en las ciudades contemporáneas, donde puede aprovechar las constantes irrupciones de lo transgresor, de lo alternativo, de lo novedoso. El incremento de las nuevas tecnologías, del comercio, de la movilidad, de la concentración del control sobre los recursos, así como los flujos de información, de inversión de capital y de trabajadores, son elementos de un escenario urbano en constante proceso de cambio. Entre los efectos que encontramos, observamos una rearticulación de las clases a partir de nuevas caracterizaciones de su identidad y de nuevas divisiones en el proceso de producción, así como una fuerte polarización social y espacial (Soja 2008: 285). 
Las "ciudades creativas", tal y como las define Florida, están repletas de episodios de gentrificación, de "acumulación por desplazamiento", de segregación residencial, de clasismo, de elitismo, de distinción. Esta formalización de estilos de vida distintivos tiene como principal labor la de ser "atractores" de inversiones, de flujos de capital, etcétera. De este modo se desarrolla todo un nicho de competencia de nuevos mercados, como los que comercian con los centros de las ciudades como espacios de multiculturalismo, de moda o de estética. Dado que "el capital tiene modos de apropiarse, de extraer los excedentes producidos por las diferencias y las variaciones culturales locales y por los significados estéticos sea cual sea el origen" (Harvey 2007: 433), su búsqueda consiste por tanto en "encontrar formas de absorber, subsumir, mercantilizar y monetizar dichas diferencias en suficiente medida como para poder apropiarse de las rentas de monopolio de las mismas".

En conclusión, podemos vincular los procesos de marketing urbano y de rehabilitación urbana con el fortalecimiento de nuevas formaciones de clase media y media-alta, de un habitus metropolitano, que emergen con la aparición de sectores en expansión de la producción en el capitalismo cognitivo. Como ya hemos explicado, los estilos de vida y consumo determinan el comportamiento colectivo y no ya solo la variable "condición económica". Por este motivo, una investigación social crítica debe indagar en la producción de estas nuevas civilidades, en sus cualidades, en su capacidad de modificar los significados del espacio y transformar las relaciones de poder establecidas, aun sin olvidar la condición eco- nómica, que supedita siempre a todas las demás. Desde luego que esta lógica, que a simple vista puede parecer natural, es potenciada por los poderes públicos, ya que es parte fundamental del proceso de reestructuración metropolitana actual. Pensar que son solo una serie de casualidades -léase la sobremodernidad, el trabajo terciarizado avanzado, los nuevos gustos y estilos de vida, o el paso de lo viejo a lo nuevo- sería no intentar complejizar en una regeneración urbana que se podría haber desarrollado de muchas otras formas.

Planteamos en definitiva que la gentrificación es más que la explotación de una renta de monopolio por parte de inversores y especuladores, ya que también comprende una serie de capitales culturales, sociales y simbólicos que condicionan la eficacia de este tipo de procesos. Así, entendemos la economía cultural urbana y el trabajador del conocimiento como dispositivo gentrificador y modo de extracción capitalista fundamental en el centro de las ciudades, basado en la articulación de la centralidad exclusiva de la ciudad posfordista como modelo de acumulación y reproducción del capital. Tras de sí, uno de los ejes vertebradores del proyecto de ciudad neoliberal: el aprovechamiento por parte de los poderes públicos de un habitus metropolitano que estas nuevas clases medias importan a estas áreas mediante sus estilos de vida y consumo distintivos. Este habitus será utilizado como recurso para hegemonizar ciertos espacios urbanos, haciéndolo ejemplarizante y provocando, por ende, "acumulación por desplazamiento" en las formas de consumo de otros sectores sociales vulnerables.

\section{NOTAS}

1 El museo, más allá de ser un contenedor de saber y conocimiento, presenta muchas trampas, ya que, bajo su condición de herramienta pedagógica, genera sentido y valor a las cosas mismas, centralizando de una manera casi ortodoxa lo que es importante culturalmente y lo que no. Entre las paredes de esta institución se construye un discurso ortodoxo del arte. Por sí mismos los museos son el elemento hegemónico e icónico de la representación artística. Es paradójico que lo que queda fuera de ellos será cuestionado como arte, pero lo que finalmente entre adquirirá un estatus que antes no poseía. Para descifrar su sentido, no hay que entenderlo como una figura física (como un edificio), sino como un sistema discursivo (Expósito 1998). El museo no es ese lugar universal y neutro, ni el arte que alberga ahistórico ni universal; no es un edifico arquitectónico aislado sino una institución social y política, que mediante su valor simbólico y patrimonial regenera el valor económico de su entorno. Dentro del museo no solo es revalorizado para el artista el valor de su producto, sino que también el prestigio de un artista puede revalorizar el prestigio del museo y, por ende, el territorio y el gobierno que lo posee.

"Mi definición del núcleo de la clase creativa incluye a las personas que se dedican a la ciencia y la ingeniería, a la arquitectura y al diseño, a la educación, al arte, y a la música y el espectáculo, y cuya función es generar nuevas ideas, nueva tecnología y/o nuevos contenidos creativos. Alrededor de este núcleo, la clase creativa también abarca a un grupo más amplio de profesionales creativos, en el mundo de la empresa y de las finanzas, en el ámbito legal y en el sanitario, y en otros campos relacionados" (Florida 2010: 47).

3 Ya Brooks (2001) parecía afinar en la descripción de los nuevos deseos y actitudes de esta clase, que describe con ciertos tintes sarcásticos en su explicación del declive de la sociedad WASP -White AngloSaxon Protestant- mediante la emergencia de un cambio social generado por una recombinación de la vieja burguesía y los valores bohemios que surgen tras los cambios culturales de los sesenta y setenta del siglo pasado.

$4 \quad$ Si bien el capital cultural, el simbólico y el social construyen un modelo para pensar el campo social y así poder posicionar a los distintos agentes en los espacios físicos, no debemos olvidar que el capital económico impone su estructura a los demás tipos de capital. 


\section{ReferenCias BibLIOgRÁficas}

Alonso, L. E. 1986. "La producción social de la necesidad". Economistas, Año 4, 18 (Ejemplar dedicado a Economía de los servicios sociales). 26-31. http://unedbarcelona.es/es/biblioteca/articulos-y-capitulos-recomendados/sociologia-1/La\%20produccion $\% 20$ social\%20 de\%20la\%20necesidad.pdf

Alonso, L. E. 2004. "Las políticas del consumo: transformaciones en el proceso de trabajo y fragmentación de los estilos de vida". Revista Española de Sociología 4 (1): 7-50.

Alonso, L. E. 2005. "El estructuralismo genético y los estilos de vida: consumo, distinción y capital simbólico en la obra de Pierre Bourdieu". http://www.unavarra.es/puresoc/pdfs/c_lecciones/LM-Alonso-consumo.PDF

Augé, M. 1992. Los "no lugares". Espacios del anonimato. Una antropología de la sobremodernidad. Barcelona: Gisa.

Bauman, Z. 2007. Modernidad líquida. Buenos Aires: Fondo de Cultura Económica.

Bianchini, F. y M. Parkinson. 1993. Cultural policy and urban regeneration: the West European experience. Manchester University Press.

Blockland, T. y G. Van Eijk. 2010. "Do People Who Like Diversity Practice Diversity in Neighbourhood Life? Neighbourhood Use and the Social Networks of "Diversity-Seekers' in a Mixed Neighbourhood in the Netherlands". Journal of Ethnic and Migration Studies 36(2): 313332. http://dx.doi.org/10.1080/13691830903387436

Bologna, S. 2006. Crisis de la clase media y posfordismo. Madrid: Akal. Cuestiones de Antagonismo.

Boschma, R. A. y M. Fritsch. 2009. "Creative class and regional growth: Empirical evidence from seven European countries". Economic Geography 85(4): 391-423. http://dx.doi.org/10.1111/j.1944-8287.2009.01048.x

Bourdieu, P. 1984/1985. "Espacio social y génesis de las clases". Espacios de crítica y producción julio/agosto 1985: 24/35. Buenos Aires: Secretaría de Bienestar Estudiantil y Extensión Universitaria, FFyL-UBA [Original publicado en Actes de la Recherche en Sciences Sociales 52/53. París, junio de 1984].

Bourdieu, P. 1988. La distinción. Criterio y bases sociales del gusto. Madrid: Taurus.

Bourdieu, P. 1999. La miseria del mundo. Madrid: Akal.

Bourdieu, P. 2000a. Esquisse d'une théorié de la pratique. París: Seuil/Points.

Bourdieu, P. 2000b. Cuestiones de Sociología. Madrid: Istmo.

Bourdieu, P. 2010. El sentido social del gusto. Elementos para una sociología de la cultura. Madrid: Siglo XXI.

Boyer, R. 1986. La théorie de la régulation: une analyse critique. La découverte.

Bridge, G. 1995. "The space for class? On class analysis in the study of gentrification". Transactions of the Institute of British Geographers 20 (2): 236-247. http://dx.doi. org/10.2307/622434

Brooks, D. 2001. Bobos en el paraíso: Ni hippies ni yuppies: un retrato de la nueva clase triunfadora. Barcelona: Grijalbo Mondadori.

Buck, N., I. Gordon, A. Harding e I. Turok. 2005. Changing cities: Rethinking competitiveness, cohesion and governance. Palgrave Publishers Limited.

Butler, T. 1997. Gentrification and the Middle Classes. Aldershot: Ashgate.

Butler, T. 2002. "Thinking Global but Acting Local: the Middle Classes in the City". Sociological Research Online 7 (3). http://www.socresonline.org.uk/7/3/butler.html
Butler, T. y G. Robson. 2001. "Social capital, gentrification and neighbourhood change in London: a comparison of three south London neighbourhoods". Urban Studies 38: 21452162. http://dx.doi.org/10.1080/00420980120087090

Carman, M. 2006. Las trampas de la Cultura. Los "intrusos" y los nuevos usos del barrio de Gardel. Buenos Aires: Paidós.

Carrillo, J. 2007. Las nuevas fábricas de la cultura. Disponible en www.medialab-prado.es/mmedia/689

Castells, M. 2001. La sociología urbana de Manuel Castells. Susser, I. (comp.). Madrid: Alianza Ensayo.

Centner, R. 2008. "Places of privilege consumption practices: spatial capital, the Dot.com habitus, and San Francisco's Internet Boom". City \& Community 7(3).

Cunningham, S. 2002. "From cultural to creative industries: theory, industry and policy implications". Media International Australia incorporating Culture and Policy, 102(1): 54-65.

De Giorgi, A. 2006. El gobierno de la excedencia: Postfordismo y control de la multitud. Madrid: Traficantes de sueños.

Deutsche, R. y C. Ryan. 1984. "The fine art of gentrification". October 31: 91-111.

Delgado, M. 2007. La ciudad mentirosa. Fraude y miseria del "Modelo Barcelona". Madrid: La Catarata.

De Nicola, A.; B. Vecchi, y G. Roggero. 2008. "Contra la clase creativa”, en Transform. Producción cultural y prácticas instituyentes. Líneas de ruptura en la crítica institucional. pp. 43-56.

Domínguez, M. 2008. "Trabajo material e inmaterial. Polémicas y conceptos inestables, marco teórico y estado de la cuestión". Youkali, Revista Crítica de las Artes y el Pensamiento, Madrid. Disponible en http://www.youkali.net/5a1-YOUKALI-Dominguez-Sanchez-Pinilla.pdf

Elias, N. 1990 [1987]. La sociedad de los individuos: ensayos. Barcelona: Península.

Elias, N. 2001. El proceso de la civilización: investigaciones sociogenéticas y psicogenéticas. México D.F.: Fondo de Cultura Económica.

Expósito, M. 1998. "Abajo los muros del museo. El arte como práctica social intramuros". Mientras tanto 72 , subido a su propio sitio: http://marceloexposito.net/pdf/exposito_abajolosmurosdelmuseo.pdf

Fainstein, S. y D. Gladstone. 1999. "Evaluating Urban Tourism". En Judd, D. y Fainstein, S. (eds.) The Tourist City: 21-34. New Haven / London: Yale University Press.

Featherstone, M. 1995. "Localism, globalism and cultural identity". En Featherstone, M., Undoing culture. Globalization, postmodernism and identity: 102-125. London: Sage Publications.

Featherstone, M. 1991. Consumer culture and postmodernism.

Florida, R. 2003. The rise of the Creative Class: and how its transforming work, leisure, community and everyday life. New York: Basic Books.

Florida, R. 2005. Cities and the creative class. Routledge.

Florida, R. 2010. La clase creativa: La transformación de la cultura del trabajo y el ocio en el siglo XXI. Barcelona: Paidós.

Fournier, J.M. 2003. "Nouvelles temporalités, changements spatiaux et inégalités sociales. L'example des villes pétrolieres du Casanare (Colombie)". Cybergeo: European Journal of Geography, Espace, Société, Territoire, document 238.

García, E. y J. Sequera. 2013. "Gentrificación en centros urbanos: Aproximación comparada a las dinámicas de Madrid y Buenos Aires". Quid 16 (3): 44-61. 
Garnham, N. 2005. "From cultural to creative industries: An analysis of the implications of the "creative industries" approach to arts and media policy making in the United Kingdom". International journal of Cultural Policy, 11(1): 15-29. http://dx.doi.org/10.1080/10286630500067606

Gill, R. y A. Pratt. 2008. "In the social factory? Immaterial labour, precariousness and cultural work". Theory, culture \& society, 25(7-8): 1-30. http://dx.doi. org/10.1177/0263276408097794

Gotham, K.2005. “Tourism Gentrification:The Case ofNew Orleans' Vieux Carre (French Quarter)". Urban Studies 42(7): 10991121. http://dx.doi.org/10.1080/00420980500120881

Hall, P. 1998. Cities in civilization. New York: Pantheon.

Harvey, D. 1989. "From managerialism to entrepreneurialism: The transformation in urban governance in late capitalism". Geografiska Annaler. Series B, Human Geography 71(1): 3-17. http://dx.doi.org/10.2307/490503

Harvey, D. 2007. Espacios del capital. Hacia una geografía crítica. Madrid: Akal.

Jacobs, J. 1969. The economy of cities. Nueva York: Random House.

Janoschka, M. y J. Sequera. 2016. "Gentrification in Latin America: addressing the politics and geographies of displacement". Urban Geography 37(8): 1-20. http:// dx.doi.org/10.1080/02723638.2015.1103995

Janoschka, M., J. Sequera y L. Salinas. 2014. "Gentrification in Spain and Latin America - a Critical Dialogue". International Journal of Urban and Regional Research 38(4): 1234-1265. http://dx.doi.org/10.1111/1468-2427.12030

Krätke, S. 2010. '“Creative Cities' and the rise of the dealer class: a critique of Richard Florida's approach to urban theory". International Journal of Urban and Regional Research 34(4): 835-853. http://dx.doi.org/10.1111/ j.1468-2427.2010.00939.x

Lang, R. y K. Danielsen. 2005. "Review roundtable: Cities and the creative class". Journal of the American Planning Association 71(2): 203-220.

Lash, S. y S. Urry. 1994. Economies of signs \& space. London: Sage Publication.

Ley, D. 2003. "Artist, aestheticisation and the field of gentrification". Urban Studies 40(12): 2527-2544. http://dx.doi. org/10.1080/0042098032000136192

Ley, D. 1996. The New Middle Class and the Remaking of the Central City. Oxford: Oxford University Press.

Lorey, I. 2008. "Gubernamentalidad y precarización de sí. Sobre la normalización de los productores y las productoras culturales". En Transform. Producción cultural y prácticas instituyentes. Líneas de ruptura en la crítica institucional: 57-78.

Marcuse, P. 1985. "Gentrification, abandonment and displacement: connections, causes and policy responses in New York City". Journal of Urban and Contemporary Law 28: 195-240.

Markusen, A. 2006. "Urban development and the politics of a creative class: evidence from a study of artists". Environment and planning A 38(10): 1921-1940. http:// dx.doi.org/10.1068/a38179

May, J. 1996. "Globalization and the politics of place and identity in an inner city London neighbourhood". Transactions of the Institute of British Geographers 21(1): 194215. http://dx.doi.org/10.2307/622933

Menger, P. M. 1999. "Artistic labor markets and careers". Annual review of sociology 25, 541-574. http://dx.doi. org/10.1146/annurev.soc.25.1.541

Mommaas, H. 2004. "Cultural clusters and the post-industrial city: towards the remapping of urban cultural policy". Urban studies 41(3): 507-532. http://dx.doi. org/10.1080/0042098042000178663

McGuigan, J. 2009. "Doing a Florida thing: the creative class thesis and cultural policy". International Journal of Cultural Policy 15(3): 291-300. http://dx.doi. org/10.1080/10286630902763281

Méndez, R., J. J. Michelini, J. Prada y J. Tébar. 2012. "Economía creativa y desarrollo urbano en España: una aproximación a sus lógicas espaciales". EURE 38(113): 5-32. http://dx.doi.org/10.4067/S025071612012000100001

Navarro, C. J. y G. Guerrero. 2010. "La relevancia de las industrias culturales en las ciudades españolas. Un primer acercamiento". En Jornadas Repensando la Metrópolis. Prácticas Experimentales en Torno a la Construcción de Nuevos Derechos Urbanos, Centro de Estudios. Andaluces, Málaga, 8 y 9 de julio.

Peck, J. 2005. "Struggling with the creative class". International Journal of Urban and Regional Research 29(4): 740:770. http://dx.doi.org/10.1111/j.1468-2427.2005.00620.x

Pendlebury, J., M. Short y A. While. 2009. "Urban World Heritage Sites and the problem of authenticity". Cities 26(6): 349-358. http://dx.doi.org/10.1016/j.cities.2009.09.003

Podmore, J. 1998. "(Re)reading the 'loft living' habitus in Montreal's inner city". International Journal of Urban and Regional Research 22: 283-302. http://dx.doi. org/10.1111/1468-2427.00140

Savage, M. J., J. Barlow, P. Dickens y T. Fielding. 1992. Property, Bureaucracy and Culture: Middle Class Formation in Contemporary Britain. Andover, MA: Routledge, Chapman and Hall.

Shaw, K. 2005a. "The Place of Alternative Culture and the Politics of its Protection in Berlin, Amsterdam and Melbourne". Planning Theory and Practice 6: 149-169. http://dx.doi.org/10.1080/14649350500136830

Scott, A. J. 2008. Social Economy of the Metropolis: Cognitive-Cultural Capitalism and the Global Resurgence of Cities: Cognitive-Cultural Capitalism and the Global Resurgence of Cities. OUP Oxford. http://dx.doi. org/10.1093/acprof:oso/9780199549306.001.0001

Scott, A. J. 2007. "Capitalism and urbanization in a new key? The cognitive-cultural dimension". Tabula Rasa, (6), 195-217. http://dx.doi.org/10.1353/sof.2007.0078

Scott, A. J. 2006. "Creative cities: Conceptual issues and policy questions". Journal of urban affairs 28(1): 1-17. http://dx.doi.org/10.1111/j.0735-2166.2006.00256.x

Scott, A. J. 2004. "Cultural-products industries and urban economic development prospects for growth and market contestation in global context". Urban affairs review 39(4): 461-490. http://dx.doi. org/10.1177/1078087403261256

Sequera, J. y M. Janoschka. 2015. "Gentrification dispositifs in the historic centre of Madrid: a re-consideration of urban governmentality and state-led urban reconfiguration". En Lees, L., Shin, H.B. \& E. López-Morales (Eds.), Global Gentrifications: Uneven Development and Displacement. Bristol: Policy Press. http://dx.doi. org/10.1332/policypress/9781447313472.003.0019

Sequera, J. 2014. "Ciudad, espacio público y gubernamentalidad neoliberal". Urban (7): 69-82.

Sequera, J. 2013. "Gentrificación en el centro histórico de Madrid: el caso de Lavapiés". En La ciudad neoliberal: Gentrificación y exclusión en Santiago de Chile, Buenos Aires, Ciudad de México y Madrid (pp. 233-255).

Slater, T. 2009. "Missing Marcuse: On gentrification and displacement". City 12(2-3): 292-311. http://dx.doi. org/10.1080/13604810902982250 
Smith, N. 1979. "Toward a theory of gentrification: a back to the city movement by capital, not people". Journal of the American Planning Association 45(4): 538-548. http://dx.doi.org/10.1080/01944367908977002

Smith, N. 1982. "Gentrification and uneven development". Economic Geography 58(2): 139-155. http://dx.doi. org/10.2307/143793

Soja, E. W. 2008. Postmetrópolis. Estudios críticos sobre las ciudades y las regiones. Madrid: Traficantes de sueños.

Storper, M., y A. J. Scott. 2009. "Rethinking human capital, creativity and urban growth". Journal of economic geography 9(2): 147-167. http://dx.doi.org/10.1093/jeg/lbn052
Yproductions 2009. Innovación en cultura. Una introducción crítica a la genealogía y usos del concepto. Madrid: Traficantes de sueños.

Yúdice, G. 2002. El recurso de la cultura: usos de la cultura en la era global. Barcelona: Gedisa.

Zukin, S. 1989. Loft Living: Culture and Capital in Urban Change. Baltimore: Johns Hopkins University Press.

Zukin, S. 1996. The cultures of cities. Wiley-Blackwell.

JORGE SEQUERA es Doctor en Sociología por la Universidad Complutense de Madrid. Actualmente es profesor en el Departamento de Análisis Social de la Universidad Carlos III de Madrid, miembro del equipo internacional de la red de investigación LXNIGHTS y cofundador de la "Oficina de Urbanismo Social" de Madrid. 